\title{
Spectral Efficiency of FBMC Over OFDM System
}

\author{
A. Abhishiktha, S. Neeraja, K.V. Satya Kumar, A. Lakshmi Narayana
}

\begin{abstract}
Significant wireless broadband technology used in various cellular standards is Orthogonal Frequency Division Multiplexing (OFDM) which will make use of Multi Carrier Modulated (MCM) systems. Even though OFDM has numerous advantages, it is hard to employ OFDM for complex networks. It is very hard to establish synchronization in mobile environments as it is difficult to predict the Doppler shifts of different users, which results in inter carrier interference (ICI). Further, filters associated with OFDM carrier have comparatively large sidebands which outcomes in Out of Band $(\mathrm{OOB})$ radiations. Insufficient spectral usage is provided by CP-OFDM by using more guard band. So the problems caused by traditional OFDM/CP-OFDM can be answered by employing a new system termed as Filter Bank Multi Carrier (FBMC) System. It is a form of MCM and it can be considered as an advanced cyclic-prefix (CP-OFDM). In OFDM, whole band gets filtered while in FBMC, each sub carrier band is independently filtered. The primary objective of this work is to relate the performance of $5 G$ modulation technique such as FBMC against OFDM and to suggest an ideal waveform for $5 G$ communication in regard to high spectral efficiency, spectral density, BER and less Peak to Average Power Ratio (PAPR).
\end{abstract}

Keywords: Filter Bank Multi Carrier (FBMC), Orthogonal Frequency Division Multiplexing (OFDM), PAPR, Spectral Efficiency.

\section{INTRODUCTION}

The elementary concept behind the Multi Carrier Modulation technique is the principle of separating the available spectrum into several sub band / sub channel and allocating a carrier to each of them and allocating the information to be sent among the sub carrier-1 sub band. Each sub carrier now has a lower bit rate. A precise choice of the basis function is acceptable for their overlapping and thus yields higher spectral efficiency. With the raise in count of sub carriers, the spectrum shape turns out to be asymptotically rectangular. Multi carrier modulation which has frequency overlapping basis functions is known as OFDM.

OFDM is the technique that is preferred ideally in point-to-point communications when coming to broadband wired and wireless channels. However, in few applications such as cognitive radios, where a subset of subcarriers is

Revised Manuscript Received on December 13, 2019.

* Correspondence Author

A. Abhishiktha*, Department of Electrical, Electronic Communication Engineering, GITAM Deemed to be University, Visakhapatnam, India. Email: aremandaabhishiktha@gmail.com

S. Neeraja, Department of Electrical, Electronics and Communication Engineering, GITAM Deemed to be University, Visakhapatnam, India. Email: neeraja.sajja@gitam.edu

K. V. Satya Kumar, Department of Electrical, Electronics and Communication Engineering, GITAM Deemed to be University, Visakhapatnam, India. Email: vsatyakumar.kudupudi@gitam.edu

A. Lakshmi Narayana, Department of Electronic Communication Engineering, Anil Neerukonda Institute of Technology \& Sciences, Visakhapatnam, India. Email: lakshminarayana.ece@anits.edu.in assigned to each user, OFDM may not be a desirable solution. OFDM system has major deficiency of large PAPR value. In this regard various PAPR reduction techniques are implemented earlier by researchers. An effective way to decrease PAPR is the unitary matrix preceded by companding. [4] The above stated issue can be further reduced by using FBMC.

In FBMC, filtering is done for each sub-carrier by changing the frequency considering the prototype filter which is formed earlier which collectively form filter bank, where as a single filter is used for all sub-carriers in OFDM. [7] 5G is intended to provide high-speed connectivity in highly reliable spectrally effective way. QAM-FBMC that totally decreases the innate overheads in CP-OFDM is planned for this purpose [3]. It enhances spectral efficiency against the CP-OFDM. So, QAM-FBMC is the best waveform solution in 5G. [3]

The spectral density of FBMC is greater than that of the OFDM which is near to the normalized frequency when related to all other $5 \mathrm{G}$ modulation techniques. It is observed that with increase in duration of bursts, the spectral efficiency of FBMC increases. [9].

\section{OFDM SYSTEM MODEL}

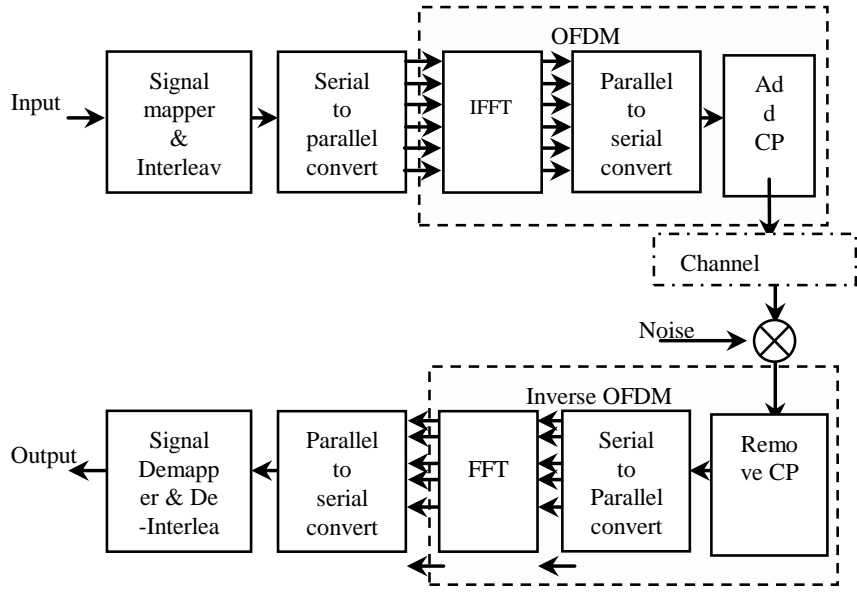

Fig. 1.OFDM block diagram

The Fig 1 shows the OFDM transceiver section in which the serial input data bits are transmitted into a parallel data with the help of serial to parallel conversion technique. Each and every parallel bit is modulated with dissimilar sub carriers. The output modulated signals (which are orthogonal to each other) are then united to form a composite signal with components of different harmonic frequency. The process of conversion from serial to parallel (followed by modulation) and from parallel to serial can be implemented with the help of IFFT.

In an OFDM system, the data made to small packets by maintaining orthogonality. This is obtained by modulating the data using QPSK. 
Then, IFFT is performed followed by parallel-to-serial converter. ISI can be reduced by adding cyclic prefix and passed through channel where noise will be added up. In Demodulation, the received data is initially passed through a low pass filter where recurring prefix will be removed. FFT is performed after the serial-to-parallel conversion. OFDM is more responsive towards Doppler shifts that disturb the offset of carrier frequency, which produce ICI that can however be reduced in the FBMC.

\section{FILTER BANK MULTICARRIER (FBMC)}

FBMC is an advancement of OFDM by getting superior in some of the issues. FBMC can use existing channel capacity in enhanced manner as the spectrum efficiency is at a higher level.

Current FBMC approach can be specified as OQAM-OFDM. Stop-band attenuation in OQAM-OFDM is maximum for a predefined filter length and amount of subcarriers. Block representation of FBMC transmitter and receiver are given below.

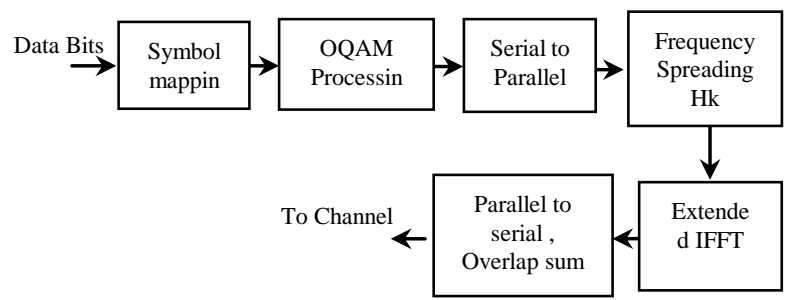

Fig. 2.Block diagram of FBMC Transmitter

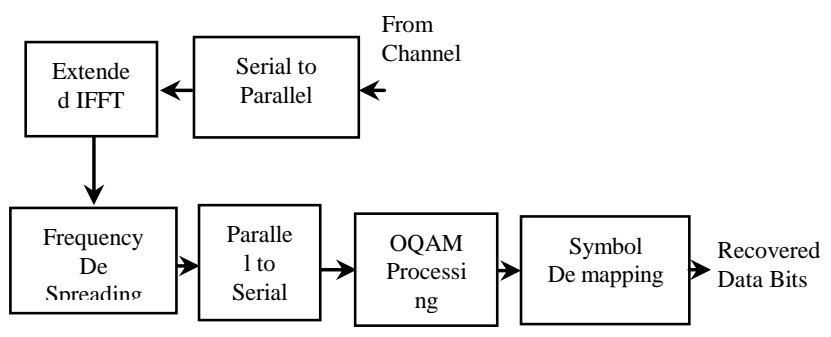

Fig. 3.Block diagram of FBMC Receiver

The FBMC base band signal for any integer $k$ can be represented as

$S_{F B M C}(k)=\sum_{n=Z} \sum_{m=0}^{M-1} a_{m, n} g\left(k-n N_{1}\right) e^{f \frac{2 \pi}{M} w k} e^{j \theta_{m, n}}$

Whereas with $\mathrm{g}$ the filter

$\mathrm{N}_{1}=\mathrm{M} / 2$ is the discrete-time offset, $\varphi_{\mathrm{m}, \mathrm{n}=} \pi / 2(\mathrm{n}+\mathrm{m})$ is the added phase term for the $\mathrm{m}$ (subcarrier) and $\mathrm{n}$ (symbol index), $\mathrm{a}_{\mathrm{m}, \mathrm{n}}$ denotes the transmitted symbols real value which is obtained from constellation of a QAM real and imaginary parts.

\section{COMPARISION OF FBMC OVER OFDM}

FBMC aims to triumph over some of the drawbacks that were encountered with OFDM. The main negative aspect in OFDM is that it requires the use of cyclic prefix which reduces the transmission throughput by wasting power.
Another drawback of OFDM is interference in unsynchronized signal and spectral leakage due to weak sub carrier spectral localization. This drawback of OFDM can be overcome by using Filter bank multicarrier. In an OFDM system as carriers gets modulated, side lobes are spread out on both the sides whereas in filter bank system, the filters remove these lobes and thus resulting in a good carrier. Systems using OFDM modulation are less complicated than FBMC. FBMC provides more selective and spectrum efficient system.

\section{PERFORMANCE PARAMETERS FROM LITERATURE \& RESULT}

Parameters like Spectral Efficiency, BER and PAPR are considered for comparing FBMC and OFDM.

\section{A. Spectral Efficiency}

The feasible bandwidth under which the bits can be transmitted successfully is represented by Spectral Density.

\section{B. BER}

BER is the Bit Error Rate in which number of bits error at the receiver when we transmitted a message i.e. due to various reasons such as noise, interference, distortion or bit synchronization. It is also called as Bit Error Ratio.

\section{SNR}

SNR shows the measure of noise (undesirable voltage levels) is included into the transmitted signal as when it is transmitted through medium.

\section{PAPR}

PAPR is the ratio of power of the sample having highest power in transmitted symbol to the normal symbol power.

\section{E. Data From Literature}

Table- I: FBMC and OFDM over various parameters

\begin{tabular}{|l|l|l|}
\hline \multicolumn{1}{|c|}{$\begin{array}{c}\text { Performance } \\
\text { parameters }\end{array}$} & OFDM & FBMC \\
\hline PAPR & High & High \\
\hline Out of band & High & Low \\
\hline CP & Yes & No \\
\hline Spectral Efficiency & Low & High \\
\hline SNR & Medium & Low \\
\hline BER & High & Low \\
\hline
\end{tabular}

PAPR can also be reduced by using precoding and companding transforms to the existing FBMC technique, which is the novel work to be done in further. 


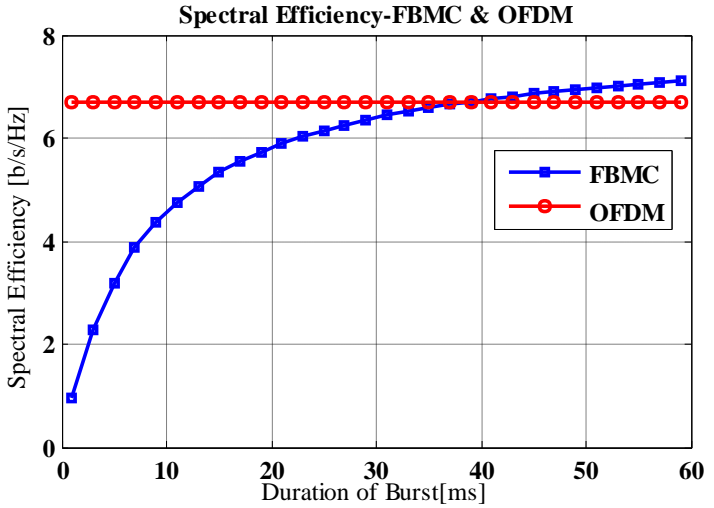

Fig. 4.Spectral Efficiency of FBMC and OFDM

Table- II: Spectral Efficiency by varying duration of bursts

\begin{tabular}{|l|l|l|l|}
\hline \multirow{2}{*}{ S.N } & \multirow{2}{*}{$\begin{array}{l}\text { Duration of } \\
\text { Burst [ms] }\end{array}$} & \multicolumn{2}{|c|}{$\begin{array}{c}\text { Spectral Efficiency } \\
\text { (b/s/Hz) }\end{array}$} \\
\cline { 3 - 4 } & & FBMC & OFDM \\
\hline 1 & 1 & 0.941 & 6.693 \\
\hline 2 & 5 & 3.2 & 6.693 \\
\hline 3 & 9 & 4.364 & 6.693 \\
\hline 4 & 13 & 5.073 & 6.693 \\
\hline 5 & 17 & 5.551 & 6.693 \\
\hline 6 & 21 & 5.895 & 6.693 \\
\hline 7 & 25 & 6.154 & 6.693 \\
\hline 8 & 30 & 6.356 & 6.693 \\
\hline 9 & 40 & 6.763 & 6.693 \\
\hline 10 & 50 & 6.974 & 6.693 \\
\hline 11 & 60 & 7.098 & 6.693 \\
\hline
\end{tabular}

It is observed from Figure 4 that the even though spectral efficiency in FBMC is increasing as the duration of burst is increased from $1 \mathrm{~ms}$ to $38 \mathrm{~ms}$, but it is below the OFDM line. It can be seen that if the duration is increased further then spectral efficiency will cross the OFDM line and further increases as the duration of bursts are increased from $39 \mathrm{~ms}$ to $60 \mathrm{~ms}$.

\section{CONCLUSION}

In this paper, the spectral analysis of FBMC over OFDM system is presented. From the results, it is observed that spectral efficiency of FBMC systems go beyond the OFDM by increasing the burst size beyond the $38 \mathrm{~ms}$. The FBMC system provides signal separation through filtering which avoids the need for perfect synchronization among signals. In FBMC the channel is subjected to the number of interfering narrow band signals which avoids the poor spectral of subcarrier signals. Although FBMC is facing some design issues such as channel estimation and PAPR but the spectral efficiency will cross the OFDM line and further increases as the duration of bursts are increased.

\section{REFERENCES}

1. Behrouz Farhang-Boroujeny, "OFDM versus Filter Bank Multicarrier", Development of Broadband Communication Systems, IEEE Signal Processing Magazine, pp. 93-112, May 2011

2. Behrouz Farhang-Boroujeny, "Filter Bank Multicarrier Modulation: A Waveform Candidate for 5G and Beyond", in Hindawi Publishing Corporation, Advanced in Electrical Engineering, Volume 2014, Article ID 482805 .

3. C. Kim, Y. H. Yun, K. Kim and J. Seol, "Introduction to QAM-FBMC: From Waveform Optimization to System Design,"in IEEE Communications Magazine, vol. 54, no. 11, pp. 66-73, November 2016.

4. R. Chandrasekhar, M. Kamaraju, M. V. S. Sairam and G. T. Rao, "PAPR reduction using combination of Precoding with Mu-Law companding technique for MIMO-OFDM systems," 2015 International Conference on Communications and Signal Processing (ICCSP), Melmaruvathur, 2015, pp. 0479-0483.

5. R. Chandrasekhar, M. Kamaraju, K. RushendraBabu, B. Ajay Kumar "Optimization of Peak to Average Power Ratio Reduction using Novel Code for OFDM Systems" Springer International Conference on Microelectronics, Electromagnetic and Telecommunication (ICMEET-2015) 18th -19th December 2015, Visakhapatnam, ISBN 978-81-322-2726-7, page numbers 267-275.

6. F. Schaich and T. Wild, "Waveform contenders for 5G - OFDM vs. FBMC vs. UFMC," Communications, Control and Signal Processing (ISCCSP), 2014 6th International Symposium on, Athens, 2014, pp. 457-460.

7. M. Bellanger. FS-FBMC: An alternative scheme for filter bank based multicarrier transmission. In 5th International Symposium on Communications Control and Signal Processing (ISCCSP), pages 1-4, May 2012

8. K. Krishna Kishore, P.Rajesh Umar, "Comprehensive Analysis of UFMC with OFDM and FBMC" in Indian Journal of Science and Technology, Vol 10 (17), May 2017

9. M. Payaro,' A. Pascual-Iserte, and M. Najar,' "Performance comparison between FBMC and OFDM in MIMO systems under channel uncertainty," in Proceedings of the European Wireless Conference (EW '10), pp. 1023-1030, April 2010.

10. T. Ihalainen, A. Ikhlef, J. Louveaux, and M. Renfors, "Channel equalization for multi-antenna FBMC/OQAM receivers," IEEE Transactions on Vehicular Technology, vol. 60, no. 5, pp. 2070- 2085, 2011

\section{AUTHORS PROFILE}

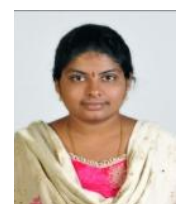

A. Abhishiktha is presently working as a SRF in the Department of Electrical, Electronics and Communications Engineering, GITAM (Deemed to be University) under DST-SERB research project. She has received the B. Tech degree from Vignan Nirula Engineering College and M.E from ANITS Engineering College, Visakhapatnam. Her areas of interests are Wireless Communications and Wireless Sensor Networks.

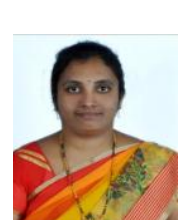

S. Neeraja is presently working as a Assistant Professor in the Department of Electrical, Electronics and Communications Engineering, GITAM (Deemed to be University). She received $\mathrm{Ph}$. D degree from Andhra university in the year 2013. She has over 14 years of Teaching Experience. She has published more than 30 research papers in various reputed International/National journals/Conferences. Her area of Interest is Wireless and Mobile Communications, Wireless Sensor Networks and CDMA/MIMO/OFDMA Wireless Communications. Currently, she is handling a DST-SERB research project.

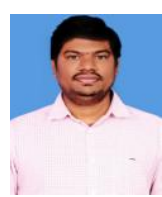

K. V. Satya Kumar is presently working as a Assistant Professor in the Department of Electrical, Electronics and Communications Engineering, GITAM (Deemed to be University). He received his M.Tech degrees from Andhra University and B.Tech from JNTU. He has over 12 years of Teaching Experience. His areas of interest are Wireless communications, Radar Signal Processing, Antennas, Digital signal processing and Image processing.

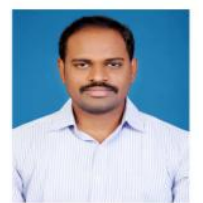

A. Lakshmi Narayana is presently working as Assistant Professor in the Department of Electronics and Communication Engineering, ANITS (Autonomous) He received $\mathrm{Ph}$. D degree from GITAM Deemed to be university in the year 2019. He has over 8 years of Teaching Experience. His area of Interest is Wireless and Mobile Communications. He has published than 10 research papers in various reputed International/National journals/Conferences in the same area. 